\section{A linear graph for digoxin radioimmunoassay}

S. E. SMITH AND ANNA RICHTER Department of Pharmacology. St. Thomas's Hospital Medical School, London SE1

The determination of drug or hormone concentrations by radioimmunoassay involves interpolation of values for radioisotope counts within standard curves, a technique which requires some dexterity in curve drawing and which results in some inaccuracy in practice. A number of linear transformations (Ekins, 1974) and computer programs (Burger et al, 1972; Duddleson et al, 1972; Livesey, 1974) for standard curves which overcome this difficulty have been described, but most of these are complex and consequently time-consuming for routine use.

In radioimmunoassays involving saturation of the antibody-binding sites a special case exists in that the bound radioactivity is directly proportional to the specific activity of ligand in the system. Thus a graph of the ratio of radioactivity bound in the absence to that in the presence of added nonradioactive ligand is linear against the concentration of added ligand (Hales and Randle, 1963). The present work, which supplements Hales and Randle's method, describes a modified expression of this relationship which has been applied to the determination of digoxin using a commercial kit. Specially constructed graph paper, which yields linearity with standard solutions, can be used directly without data transformation.

\section{Theory}

Two assumptions are made:

1. Binding of ligand (digoxin) by the antibody does not vary with the total concentration of that ligand. 2. Antibody binds radioactive and non-radioactive ligand with equal affinity and in proportion to their relative concentrations.

From these assumptions it is deduced that binding of radioactive ligand depends directly on the proportion of radioactive to total ligand (ie, specific activity) in the system. Therefore:

Radioactivity of bound ligand $=k\left[\frac{s}{\mathrm{VC}+\mathrm{s}}\right]$

where $s=$ amount of radioactive ligand, $C=$ concentration of non-radioactive ligand in the solution added, and $v=$ volume of that added

Received for publication 7 April 1975. solution. Therefore, a graphic plot of bound radioactivity against the specific activity function $\left[\frac{\mathrm{s}}{\mathrm{vC}+\mathrm{s}}\right]$ should be linear.

\section{Materials and Method}

All determinations were done with the Lanoxitest $\beta$ digoxin radioimmunoassay kit (Wellcome Reagents Ltd) in conformity with the instructions provided. In this system $\mathrm{s}=0.5 \mathrm{ng}$ and is constant between different batches, $v=0.2 \mathrm{ml}$, and $\mathrm{C}$ varied in the range 0 to $8 \mathrm{ng} / \mathrm{ml}$. Antibody-bound radioactivity was counted in a Beckman LS-100 liquid scintillation spectrometer.

\section{Results and Comment}

Within the concentration range likely to be found clinically $(\mathrm{C}=0.5$ to $8 \mathrm{ng} / \mathrm{ml})$ the relationship between bound radioactivity and the specific activity function is linear (figure), as deduced theoretically.

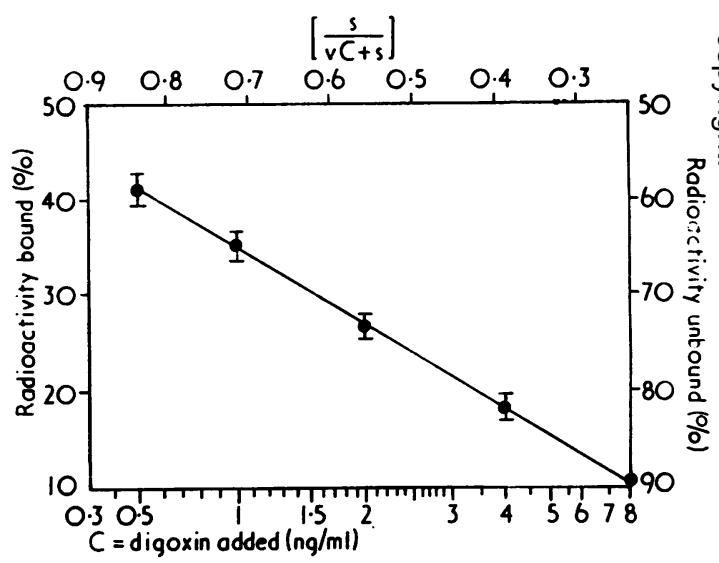

Figure Digoxin radioimmunoassay. Determination of standard solutions. Vertical lines represent $S D$ (for $C=0.5-4, n=24$; for $C=8, n=6$ ). See text for explanation of scales.

In this figure the specific activity function appears as the upper arithmetical horizontal scale; corresponding values of $\mathbf{C}$, individually calculated, appear on the lower scale opposite the appropriate values of the upper scale. Graph paper with delineation conforming to the concentration scale has been in experimental use in this laboratory for over six months. On each of approximately $\mathbf{5 0}$ occasions standard solutions have yielded satisfactory linearity. 
The use of this graph paper requires no data processing and is therefore somewhat simpler than the ratio method described by Hales and Randle (1963), as well as more convenient and more accurate than the conventional curve-fitting procedure.

Calculation of the total quantities of digoxin bound by the antibody reveals that binding was approximately constant through the range of concentrations tested, as expected from the linearity of the graph shown in the figure. With increasing concentration, however, binding declined slightly, which suggests that it may be inhibited at particularly high concentrations of ligand. The reason for this is unknown at present.

We thank St. Thomas's Hospital Endowment Fund for financial support.

\section{References}

Burger, H. G., Lee, V. W. K., and Rennie, G. C. (1972). A generalized computer program for the treatment of data from competitive protein-binding assays including radioimmunoassays. J. Lab. clin. Med., 80, 302-312.

Duddleson, W. G., Midgley, A. R., and Niswender, G. D. (1972). Computer program sequence for analysis and summary of radioimmunoassay data. Comput. biomed. Res., 5, 205-217.

Ekins, R. P. (1974). Radioimmunoassay and saturation analysis: basic principles and theory. Brit. med. Bull., 30, 3-11.

Hales, C. N. and Randle, P. J. (1963). Immunoassay of insulin with insulin-antibody precipitate. Biochem. J., 88, 137-146.

Livesey, J. H. (1974). Computation of radioimmunoassay data using segmentally linearized standard curves. Comput. biomed. Res., 7, 7-20.

\section{Book reviews}

Immunobiology of Transplantation: The Histocompatibility Systems. Edited by Fritz H. Bach. (Pp. xiii + 385; illustrated; £11.90.) New York and London: Grune and Stratton 1974.

This is the hard-cover edition (2lb 4oz) of the Proceedings of the Second International Symposium on the Cellular Aspects of Transplantation held in France in June 1973. The Proceedings were originally published in December 1973 as part of the soft-back quarterly journal Transplantation Proceedings (2lb 9oz). The papers are concerned with the parallel developments in the unravelling of the complexity of the histocompatibility systems in man and mouse. To read it all is quite hard work because there is inevitably some repetition but the acknowledged experts in this field are all represented.

The first part of the volume is concerned with the recognition phase, that is, how cells recognize the foreignness of others. The second part is devoted to the destruction phase which involves the development, in the mixed lymphocyte culture reaction, of cytotoxic lymphocytes which subsequently react against specific target cells. The third part deals with the application of these systems to the in vivo situation in man and other animals. The importance of both serological and tissue culture matching procedures is demonstrated.

The mixed lymphocyte culture reaction has applications in tissue transplantation in man, and further knowledge of the genetics which control the recognition and response phenomena of the system will undoubtedly lead to practical advances. The approach that is being used in man is to attempt to find genetically homozygous individuals by testing the offspring of first-cousin marriages.

The last part of the book deals with the question of how the antigens of the various histocompatibility systems are expressed on the lymphocyte surface. This is the area from which the next advances are likely to come.

SYLVIA D. LAWLER

Histopathological Stains and their Diagnostic Uses. By John D. Bancroft and Alan Stevens. (Pp. 149; illustrated; $£ 4 \cdot 50$.) Edinburgh, London and New York: Churchill Livingstone. 1975.

This differs from other technical manuals of its kind in that it is thinner and better illustrated and concentrates upon staining methods in histopathology. It is designed for histopathologists and their technologists with the expressed intention of trying to bring them closer together; in addition, there is emphasis on the explanation of the rationale of staining reactions. All these are admirable objectives, especially at the present time, when more and more technological training in histopathology is conducted outside the hospital laboratory. The young patholo- gist might hope that this book will tell him which are the best stains for a particuła tissue component, but the tables areging the classical form, showing the tisłue components that are demonstrated क्षेंs each staining technique. Despite the attempt to explain the underlying pring ciples of histological staining methods $\frac{\text { D }}{\mathbb{D}}$ the known chemical reactions are noe emphasized or are not stated in chemica $\vec{b}$ terms; this seems a pity when the progress of histopathological technique from an art to a science must depend upon all of us knowing the details of the chemical. mechanisms of the staining reactions which we do understand. This book mays be of particular value to the juniop. pathologist in his first year in histo pathology and to the university graduate who is making the transition from pures science into the technological aspects of histopathology.

R. A. B. DRURY

U

Automation in Microbiology and Immun : ology. Edited by Carl-Göran Hedén and Tibor Illeni. (Pp. xxvi + 610; illus trated; £18.50.) New York: London, Sydney, and Toronto: John Wiley.

An international symposium 'Rapi£ Methods and Automation in Micro biology' was held in June 1973 in Stock holm, sponsored by UNESCO, WHO, anफ़् the International Organization for Bio $\Omega$ technology and Bioengineering. Thi 